\title{
Importance de l'étape pré-analytique en spermiologie pour le diagnostic et l'évaluation des thérapeutiques
}

\author{
A. CLAVERT 1 , A. BOURGUIGNAT 2 , C. CRANZ 1
}

1 CECOS Alsace, Laboratoire de Biologie de la Reproduction, Hôpitaux Universitaires de Strasbourg, Strasbourg ; 2 Laboratoire de Biochimie Clinique, Faculté de Pharmacie, Université Louis Pasteur, Illkirch.

\section{RÉSUMÉ}

Le sperme présente une variabilité importante liée en partie à la mécanique de l'éjaculation. Il est par contre possible de réduire cette variabilité en prenant quelques précautions comme éliminer l'existence de facteurs externes par un interrogatoire, imposer un délai d'abstinence entre 3 et 5 jours, expliquer les mesures d'hygiène avant le prélèvement, vérifier si le prélèvement est complet, de veiller au maintien de l'échantillon à $37^{\circ} \mathrm{C}$, enfin bien l'homogénéiser pour obtenir une liquéfaction dans de bonnes conditions.

Mots clés : Spermogramme, abstinence, asepsie, chaleur, stress, homogénéisation

La variabilité du sperme a été magistralement mise en évidence par l'observation de Paulsen [11]. L'évolution de la concentration du sperme, d'un donneur ne présentant pas de pathologie particulière, pendant 120 semaines montre que cette valeur peut varier de 5 à 170 millions de spermatozoïdes. Si des hommes ayant une variabilité identique, sont inclus dans un protocole expérimental, il devient pratiquement impossible de mettre en évidence les effets d'une thérapeutique.
Cette variabilité observée est en fait la somme des variabilités liées à la mécanique de l'éjaculation proprement dit, mais également aux conditions pré-analytiques (état du patient au moment du prélèvement, technique de prélèvement, traitement de l'échantillon avant l'analyse) et aux facteurs de variation inhérents à la technique d'analyse utilisée.

La spermoculture est un examen déterminant pour l'instauration d'une thérapeutique antibiotique. L'étape pré-analytique peut jouer un rôle important comme le montre l'étude de Boucher et coll. [2] ; le seul fait d'expliquer les mesures d'hygiène au patient avant le prélèvement fait passer les spermocultures positives de $26,9 \%$ pour les prélèvements après conseils écrits à $13,5 \%$ pour les prélèvements après conseils transmis par oral. Comment dans ces conditions mettre en évidence un effet statistiquement positif d'une thérapeutique si les conditions d'hygiène lors du prélèvement ne sont pas strictement transmises et contrôlées suivant un protocole rigoureusement respecté.

Le contrôle des conditions pré-analytiques permet de conférer à l'analyse proprement dit une

Correspondance : A. Clavert, CECOS Alsace, Laboratoire de Biologie de la Reproduction. Hôpitaux Universitaires de Strasbourg, 67091 Strasbourg Cedex

Communication aux 3èmes Journées Nationales Assurance de Qualité en Biologie de la Reproduction, 26 \& 27 Octobre 2000, Nancy. 
puissance diagnostique bien supérieure et cela sans modifier les conditions de l'analyse.

Il est possible de diviser les différentes étapes pré-analytiques en trois parties :

- L'état dans lequel le patient arrive au laboratoire, sa condition physique.

- Le prélèvement.

- Le traitement de l'échantillon après le prélèvement et avant l'analyse.

\section{CONDITION PHYSIQUE DU PATIENT}

Le délai d'abstinence sexuelle joue un rôle des plus importants sur les paramètres du sperme. Schwartz et coll. [13] ont étudié les conséquences du délai d'abstinence sur les différents paramètres du spermogramme des donneurs de sperme. Ils ont pu évaluer les conséquences de l'allongement du délai sur le volume, la concentration, le nombre total de spermatozoïdes ainsi que la tératospermie et la mobilité. Pour le volume par exemple, le passage d'un délai de 1 à 5 jours entraîne le doublement de la valeur de cette variable. Par contre pour la tératospermie le délai n'intervient pas.

L'exposition aiguë à la chaleur, que ce soit une fièvre, une insolation ou un sauna, détermine des modifications de la mobilité.

Les substances toxiques comme médicaments, drogues, dopants et/ou toxiques professionnels peuvent également avoir des effets.

Le stress, peut totalement modifier tous les paramètres du sperme, comme le montre, la comparaison des spermogrammes d'hommes présents à Kobe lors du tremblement de terre, de ceux qui étaient restés loin de l'épicentre [7].

Le moment du prélèvement intervient car il existe des variations diurnes [3], saisonnière et environnementales qui peuvent être importantes [10].

L'interrogatoire préalable (annexe 1) permet de retrouver l'existence de facteurs externes et comportementaux qui peuvent être responsables de modifications transitoires de paramètres du spermogramme. Signaler leur existence permet de ne pas prendre de décisions thérapeutiques intempestives.

\section{CONDITIONS DU PRÉLÈVEMENT}

Les échecs de prélèvement sont rares [5, 9] si certaines précautions sont respectées.

L'accueil au téléphone et au secrétariat est déterminant. Lors de la prise de rendez-vous, des indications sur le délai d'abstinence, sur le mode de prélèvement peuvent être données, ce qui a pour effet non seulement de réduire certains facteurs de variation pré-analytique mais également de répondre à quelques questions. Ainsi l'anxiété du patient se réduit et les échecs de prélèvement sont plus rares.

Les locaux n'ont pas besoin d'être spacieux, mais il faut de quoi s'allonger et un lavabo, en position adaptée pour que le patient puisse se laver correctement les mains et la verge. Attention, ce local est considéré comme contaminé et doit être désinfecté entre deux patients. (Guide de bonnes pratiques en AMP).

Les mesures d'hygiène [12], il existe un consensus pour proposer cette procédure :

- Faire uriner au préalable.

- Lavage des mains avec un savon chirurgical.

- Lavage de la verge, en précisant bien, de rabattre le prépuce pour bien nettoyer le sillon balano-préputial.

- Essuyer avec une compresse stérile

- Expliquer ces mesures au patient et justifier leur utilité pour le diagnostic.

Le mode de prélèvement est en général la masturbation. Les études de Freund [6] ont montré qu'il n'existe pas de différence significative entre les spermes prélevés par masturbation ou par coït interrompu. Le consensus est d'imposer la masturbation, si cela n'est pas possible, il convient de bien noter le procédé effectivement utilisé. S'il s'agit d'un coït interrompu, le sperme peut être pollué par des sécrétions vaginales.

Le réceptacle doit être stérile et adapté aux conditions de prélèvement [9]. Le commerce met à notre disposition plusieurs modèles bien adaptés. Il faut faire attention aux graduations des tubes qui, dans certains cas, ne sont pas rigoureuses et demander au fabricant ses normes ou vérifier les lots. 
Le transport du prélèvement du local de prélèvement au laboratoire, doit se faire le plus rapidement possible (important pour l'évaluation du temps de liquéfaction) tout en tenant compte des conditions d'hygiène.

La perte d'une partie de l'éjaculat est possible au moment du prélèvement. Il convient d'interroger le patient sur le déroulement du prélèvement. Cela permet d'éliminer les éjaculats incomplets qui ne peuvent être analysés.

\section{CONDITIONS D'ÉVALUATION DES PARAMÈTRES PHYSICO- CHIMIQUE DU SPERME}

L'homogénéisation de l'éjaculat est indispensable avant de débuter toute étude. En effet au moment de l'éjaculation, les sécrétions sont expulsées les unes après les autres, ainsi le sperme à l'éjaculation est hétérogène.

La liquéfaction, étape physiologique dépendant de la concentration en enzymes prostatiques [4], sera dépendante de la température. Ainsi l'échantillon doit rapidement être mis à l'étuve si l'on veut étudier le temps de liquéfaction à une température de $37^{\circ} \mathrm{C}$.

Le volume peut être évalué dans le tube de prélèvement à la condition qu'un contrôle soit réalisé ou que des garanties soient données par le constructeur sur la précision des graduations, sinon une évaluation à la pipette ou par pesée doit être réalisée.

Le pH peut être évalué grâce à l'utilisation de papiers $\mathrm{pH}$, à la condition que ce papier ne soit pas stocké et qu'un contrôle soit fait avant l'utilisation d'un lot.

\section{CONDITIONS D'ÉVALUATION DE LA MOBILITÉ}

La mobilité du spermatozoïde est très dépendante de la température, température au moment de la lecture mais également de la température à laquelle est conservé l'échantillon dans l'intervalle entre l'éjaculation et la lecture, ainsi il est bon pour pouvoir bien maîtriser ce facteur de maintenir l'éjaculat toujours à $37^{\circ} \mathrm{C}$ (étuve, platine chauffante) [1]

\section{CONCLUSION}

Le respect de quelques règles pratiques quant au prélèvement et au traitement de l'échantillon avant l'analyse permet d'éviter d'augmenter la variabilité d'un examen qui présente déjà une variabilité importante liée aux méthodologies employées.

Un facteur de variabilité important est l'état physiologique du patient au moment du prélèvement, un interrogatoire rapide peut révéler des situations passagères peut et ainsi éviter des erreurs d'interprétations.

\section{RÉFÉRENCES}

1. AUGER J., JOUANNET P, : Manuel de laboratoire de l'OMS. Analyse du sperme humain et de l'interaction des spermatozoïdes avec le mucus cervical. Les éditions INSERM. 1993.

2. BOUCHER P., LEJEUNE H., PINATEL M.C., GILLE Y. : Spermoculture: improvement of the bacteriological quality of samples by direct verbal counseling before semen collection. Fertil. Steril., 1995, 64 : 657660 .

3. CAGNACCI A., MAXIA N., VOLPE A. : Diurnal variation of semen quality in human males. Hum. Reprod., 1999, 14 : 106-109.

4. CLAVERT A., CRANZ C., VIGNON F. : Les fonctions du plasma séminal. In : Les voies séminales. Dadoune, Clavert, Arvis eds. Doin, 1993, 131-136.

5. CZYBA J.C. : La masturbation au laboratoire. Andrologie, 1991, 1 : 34-35.

6. FREUND M. : Interrelationship among the characteristics of human semen and factors affecting semen specimen quality. J. Reprod. Fertil., 1982, 4 : 143-159.

7. FUKUDA M., FUKUDA K., SHIMIZU T., YOMURA W., SHIMIZU S. : Kobe earthquake and reduced sperm motility. Hum. Reprod., 1996, 6 : 1244-1246.

8. JOUANNET P. : Exploration du testicule exocrine. In: Médecine de la reproduction. P. Mauvais-Jarvis, ed. Flammarion, 1984, 211-228.

9. JOUANNET P., DAVID G. : Conditions matérielles et psychologiques du recueil du sperme. J. Gynecol. Obstét. Biol. Reprod. 1977, 6 : 55-64.

10. OSSENBÜHN S. : Exogenous influence on human fertility: fluctuation in sperm parameters and results of in-vitro fertilization coincide with conceptions in the normal population. Hum. Reprod., 1998, 13 : 2165-2171.

11. PAULSEN C.A. : In : O.M.S.: Laboratory manual for the examination of human semen and semen-cervical mucus interaction. Press Concern, Singapore, 1980, 8. 
12. PINATEL M.C., BOUCHER P., GILLE Y., PINATEL F. : Proposition d'un protocole de prélèvement pour spermoculture. Andrologie, 1994, $2: 228-233$.

13. SCHWARTZ D., LAPLANCHE A., JOUANNET P., DAVID G. : Within subject variability of human semen in regard to sperm count, volume, total number of spermatozoa and length of abstinence. J. Reprod. Fertil. 1979, $57: 391-395$.

14. SPIRA A. : Epidemiologic aspect of the relationship between temperature and male reproduction. In: Temperature and environmental effects on the testis. Zorgniotti A.W. ed., Plenum Press, 1991, 49-58.

\begin{abstract}
Importance of the pre-analytical step for diagnosis and therapeutic evaluations in spermiology.
\end{abstract}

A. CLAVERT, A. BOURGUIGNAT, C. CRANZ

Semen analysis is subject to great variability, partly due to the mechanism of ejaculation. This variability can be reduced by taking certain precautions, such as interviewing the patient to eliminate any external factor of variability, by imposing a 3- to 5-day period of sexual abstinence, by explaining the procedures to avoid bacterial contamination of the sample, by verifying whether the whole semen sample has been collected, by checking that the temperature of the sample is maintained at $37^{\circ} \mathrm{C}$ and finally, by carefully homogenising the sample to obtain good quality liquefaction.

Key words: Semen analysis, abstinence, fever, stress, homogenisation 


\section{Proposition d'interrogatoire faite par Jean MENOTTI}

(Mémoire du DIU de Biologie Appliquée à la Procréation. Besançon, Nancy, Strasbourg et Reims 1999)

- Quelle est votre profession?

- Etes-vous ou avez-vous été exposé à des substances toxiques ? à des métaux lourds ? à des pesticides ? à des irradiations?

- Etes-vous régulièrement exposé à une chaleur excessive ?

- Combien de temps passez-vous dans une voiture quotidiennement?

- Vous arrive-t-il de prendre des saunas?

- Fumez-vous ? Si oui, combien de cigarettes par jour? depuis combien de temps?

- Combien de verres de vin buvez-vous par semaine ? de bière ? de digestifs, de schnaps, d'eau-de-vie, de whisky, de rhum, de cognac...?

- Vous arrive-t-il de fumer des “joints", de la marijuana ? Si oui, combien de fois par semaine?

- Vous arrive-t-il de consommer des " drogues"?

- Vous arrive-t-il de vous doper?

- Quelle est la fréquence habituelle de vos éjaculations ? avec pénétration?

- Vous arrive-t-il d'être confronté à des troubles de l'érection ? à des éjaculations prématurées?

- Avez-vous déjà été atteint de maladies sexuellement transmissibles ?

- Avez-vous déjà ressenti des brûlures au niveau des organes génitaux ?

- Avez-vous subi un traumatisme testiculaire ou une torsion testiculaire? une intervention chirurgicale au niveau des organes génitaux ? au niveau du petit bassin ? Avez-vous été sondé ? Pendant combien de temps?

- Avez-vous été traité pour un cancer ou une maladie du sang?

- Avez-vous subi une greffe d'organe ou de moelle osseuse?

- Prenez-vous des médicaments contre les ulcères ? contre la goutte ? contre les crises d'épilepsies? contre l'hypertension artérielle? contre l'insuffisance cardiaque ? contre les crises d'angor? contre la dépression?

- Prenez-vous des antibiotiques ? des anti-viraux? des corticoïdes ? des stéroïdes anabolisants? des immunosuppresseurs? des diurétiques ? des ß-bloquants ? des neuroleptiques? Prenez-vous d'autres médicaments? 